\title{
Renal function estimation and Cockcroft-Gault formulas for predicting cardiovascular mortality in population- based, cardiovascular risk, heart failure and post-myocardial infarction cohorts: The Heart 'OMics' in AGEing (HOMAGE) and the high-risk myocardial infarction database initiatives
}

João Pedro Ferreira', Nicolas Girerd ${ }^{1}$, Pierpaolo Pellicori ${ }^{2}$, Kevin Duarte ${ }^{3,4,5}$, Sophie Girerd ${ }^{1}$, Marc A. Pfeffer ${ }^{6}$, John J. V. McMurray ${ }^{7}$, Bertram Pitt ${ }^{8,9}$, Kenneth Dickstein ${ }^{10}$, Lotte Jacobs ${ }^{11}$, Jan A. Staessen ${ }^{11}$, Javed Butler ${ }^{12}$, Roberto Latini ${ }^{13}$, Serge Masson ${ }^{13}$, Alexandre Mebazaa ${ }^{14}$, Hans Peter Brunner-La Rocca $^{15}$, Christian Delles ${ }^{7}$, Stephane Heymans ${ }^{16}$, Naveed Sattar ${ }^{7}$, J. Wouter Jukema ${ }^{17,18}$, John G. Cleland ${ }^{19}$, Faiez Zannad ${ }^{1}$, Patrick Rossignol ${ }^{1,20^{*}}$ and for the Heart 'OMics' in AGEing (HOMAGE) initiative and the High-Risk Myocardial Infarction database initiative

\begin{abstract}
Background: Renal impairment is a major risk factor for mortality in various populations. Three formulas are frequently used to assess both glomerular filtration rate (eGFR) or creatinine clearance ( $\mathrm{CrCl}$ ) and mortality prediction: body surface area adjusted-Cockcroft-Gault (CG-BSA), Modification of Diet in Renal Disease Study (MDRD4), and the Chronic Kidney Disease Epidemiology Collaboration (CKD-EPI) equation. The CKD-EPI is the most accurate eGFR estimator as compared to a "gold-standard"; however, which of the latter is the best formula to assess prognosis remains to be clarified. This study aimed to compare the prognostic value of these formulas in predicting the risk of cardiovascular mortality (CVM) in population-based, cardiovascular risk, heart failure (HF) and post-myocardial infarction (MI) cohorts.

(Continued on next page)
\end{abstract}

\footnotetext{
* Correspondence: p.rossignol@chru-nancy.fr

'INSERM, Centre d'Investigations Cliniques Plurithématique 1433, INSERM

U1116, Université de Lorraine, CHRU de Nancy, F-CRIN INI-CRCT, Nancy,

France

${ }^{20}$ Centre d'Investigations Cliniques-INSERM CHU de Nancy, Institut Lorrain du Cœur et des Vaisseaux Louis Mathieu, 4 Rue du Morvan, 54500 Vandoeuvre lès Nancy, France

Full list of author information is available at the end of the article
} 
(Continued from previous page)

Methods: Two previously published cohorts of pooled patient data derived from the partners involved in the HOMAGE-consortium and from four clinical trials - CAPRICORN, EPHESUS, OPTIMAAL and VALIANT - the high risk MI initiative, were used. A total of 54,111 patients were included in the present analysis: 2644 from populationbased cohorts; 20,895 from cardiovascular risk cohorts; 1801 from heart failure cohorts; and 28,771 from postmyocardial infarction cohorts. Participants were patients enrolled in the respective cohorts and trials. The primary outcome was CVM.

Results: All formulas were strongly and independently associated with CVM. Lower eGFR/CrCl was associated with increasing CVM rates for values below $60 \mathrm{~mL} / \mathrm{min} / \mathrm{m}^{2}$. Categorical renal function stages diverged in a more pronounced manner with the CG-BSA formula in all populations (higher $x^{2}$ values), with lower stages showing stronger associations. The discriminative improvement driven by the CG-BSA formula was superior to that of MDRD4 and CKD-EPI, but remained low overall (increase in C-index ranging from 0.5 to 2\%) while not statistically significant in population-based cohorts. The integrated discrimination improvement and net reclassification improvement were higher $(P<0.05)$ for the CG-BSA formula compared to MDRD4 and CKD-EPI in CV risk, HF and post-MI cohorts, but not in population-based cohorts. The CKD-EPI formula was superior overall to MDRD4.

Conclusions: The CG-BSA formula was slightly more accurate in predicting CVM in CV risk, HF, and post-MI cohorts (but not in population-based cohorts). However, the CG-BSA discriminative improvement was globally low compared to MDRD4 and especially CKD-EPI, the latter offering the best compromise between renal function estimation and CVM prediction.

Keywords: Population based, Cardiovascular risk, Heart failure and post-myocardial infarction cohorts, Renal function, Glomerular filtration rate formulas, Cardiovascular mortality prediction

\section{Background}

Renal impairment is a major risk factor for mortality in various populations [1-3], making it an essential risk stratification tool. In certain high-risk populations, renal function is of paramount importance for prognostic purposes in order to establish better and personalized follow-up programs and prognosis-modifying interventions [4]. Hence, risk prediction properties should be considered at least as valuable as the accuracy of renal function quantification per se. Renal function estimation in daily practice is performed by "indirect" parameters (as most accurate gold-standard methods are not practical nor economically suitable for routine use [5]), incorporating variables such as creatinine, age, gender, weight and height through a variety of available formulas. The most commonly used formulas to estimate glomerular filtration rate (eGFR) include the Modification of Diet in Renal Disease (MDRD) formula [6] and the simplified MDRD4 formula [7], both of which have been tested in diverse populations with reproducible results [8], as well as the Chronic Kidney Disease Epidemiology Collaboration (CKD-EPI) equation, which provides the most accurate GFR estimation (compared to a renal-clearance "gold standard") and is the formula advocated by contemporary consensus $[4,9,10]$. Creatinine clearance $(\mathrm{CrCl})$ estimation can be performed by the Cockcroft-Gault (CG) formula [11]. It should be emphasized that $\mathrm{CrCl}$ estimation by the $\mathrm{CG}$ formula comprises glomerular $\mathrm{CrCl}$ plus tubular $\mathrm{CrCl}$, and therefore the overall $\mathrm{CrCl}$ may overestimate GFR by up to $40 \%$ in younger individuals without chronic kidney disease $[12,13]$. However, in older individuals, the CG formula may underestimate GFR [14, 15]. The CG formula has more recently been modified and validated taking into account body surface area (BSA) [16]. This BSA-adjusted CG formula (CG-BSA) is likely to provide a more accurate estimation of $\mathrm{CrCl}$ compared to the original $\mathrm{CG}$ formula (hence the use of the CG-BSA formula in the main analysis of this manuscript) $[5,10,16,17]$.

The primary goal of a renal function estimation equation is to estimate GFR (and not tubular clearance). Therefore, for this purpose, the MDRD and CKD-EPI equations are suitable, whereas the CG/CGBSA equations are not. Nonetheless, in addition to the GFR estimation, prognostic ability is also of utmost importance. Therefore, the aforementioned equations have also been compared regarding their prognostic implications in population-based, cardiovascular $(\mathrm{CV})$ risk, heart failure $(\mathrm{HF})$, and myocardial infarction (MI) populations [3, 18-23]. While extensive information has been provided in these settings using the MDRD4 and CKD-EPI formulas for prognostic and risk estimation purposes with heterogeneous results [19-21], little is known regarding the use of the CG-BSA formula.

The aim of the present study was to conduct a headto-head comparison of the eGFR and $\mathrm{CrCl}$ formulas in terms of prognostic value in four large populations/ 
cohorts, namely population-based, CV risk, HF, and post-MI cohorts.

\section{Methods}

Study population: the Heart 'OMics' in AGEing (HOMAGE) initiative and the high-risk myocardial infarction database initiative

Eligible studies included population studies, patient cohorts, and randomized controlled trials (RCTs). All studies had baseline information on demographic, clinical, and laboratory characteristics and subsequent follow-up reports (cardiovascular mortality was used for the present study). The partners involved in the HOMAGE-consortium contributed data from completed and ongoing studies. These data have been previously published [24] and include patient data derived from (1) population-based cohorts (FLEMENGHO [25] and PREDICTOR [26]); (2) CV risk factor cohorts (ASCOT [27], PROSPER [28], HVC, DYDA [29] and BIOMARCOEURS [30]); and (3) HF cohorts (HULL LIFELAB and TIME-CHF [31]), all of which had baseline information regarding age, sex, weight, height, and plasma creatinine [24].

The High-risk MI Initiative consists of a previously published cohort of pooled patient data derived from four clinical trials [32]. Briefly, the main objectives of the project are to provide a comprehensive and statistically robust description of long-term clinical outcomes in high-risk survivors of MI and to identify the predictors of these different outcomes. In addition, the creation of this large pooled dataset provides statistical power to examine outcomes in important sub-groups of patients defined by co-morbidity and other baseline variables. The datasets included in this pooling initiative were the effect of Carvedilol on Outcome after Myocardial Infarction in Patients with Left Ventricular Dysfunction trial (CAPRICORN) [33, 34], the Eplerenone PostAcute Myocardial Infarction Heart Failure Efficacy and Survival Study (EPHESUS) [35, 36], the Optimal Trial in Myocardial Infarction with Angiotensin II Antagonist Losartan (OPTIMAAL) [37, 38], and the Valsartan in Acute Myocardial Infarction trial (VALIANT) $[39,40]$. Full details of total enrolled patients, the inclusion and exclusion criteria for each trial, the endpoints, and the results have been previously published. Each trial enrolled patients with left ventricular systolic dysfunction, HF, or both between $12 \mathrm{~h}$ and 21 days after acute MI.

The respective chairpersons of the Steering Committees of the four trials initiated the pooling project.

The studies were all conducted in accordance with the Declaration of Helsinki and approved by site ethics committees. All participants gave written informed consent to participate in the studies.
Renal function analyses and estimation of GFR and $\mathrm{CrCl}$ Baseline laboratory measurements were obtained at the time of inclusion and plasma creatinine concentrations were recorded in all studies. The eGFR and $\mathrm{CrCl}$ were calculated with the published equations for CKD-EPI [4], the four-variable MDRD4 [6], and CG-BSA [16].

\section{CKD-EPI}

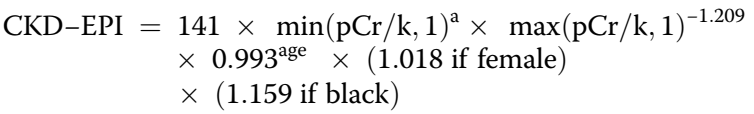

where adjusted serum creatinine was calculated as described below, $\mathrm{k}$ is 0.7 for females and 0.9 for males, a is -0.329 for females and -0.411 for males, min indicates the minimum of serum creatinine adjusted $/ \mathrm{k}$ or 1 , and max indicates the maximum of adjusted serum creatinine/ $\mathrm{k}$ or $\mathrm{l}$.

MDRD4

$$
\begin{aligned}
\text { MDRD4 }= & 186 \times \mathrm{pCr}^{-1.154} \times \mathrm{age}^{-0.203} \\
& \times(0.742 \text { if female }) \times(1.210 \text { if black })
\end{aligned}
$$

\section{CG-BSA}

$$
\begin{aligned}
\text { CG-BSA }= & {[(140-\text { age }) \times \text { weight in } \mathrm{kg} \times(0.85 \text { if female })] } \\
& \div(72 \times \mathrm{pCr} \text { in } \mathrm{mg} / \mathrm{dL})=\text { Cockcroft }- \text { Gault }
\end{aligned}
$$

followed by $\left(1.73 \mathrm{~m}^{2} \times\right.$ GFR-CG)/BSA of the patient; where the GFR-CG is in milliliters per minute and the BSA is calculated in meters squared using the Mosteller equation [41].

Patients were also classified into eGFR categories 1-5: Stage 1, eGFR $\geq 90$; Stage 2, eGFR $\geq 60$ and $<90$; Stage 3a, eGFR $\geq 45$ and $<60$; Stage $3 b$, eGFR $\geq 30$ and $<45$; Stage 4 , eGFR $\geq 15$ and $<30$; Stage 5 , eGFR $<15$.

\section{Outcomes}

The present study analyzed cardiovascular mortality (CVM) as the primary outcome. Endpoints were independently adjudicated in the respective trials and cohorts.

\section{Statistical methods}

In descriptive analyses, continuous variables are expressed as mean \pm standard deviation (SD) if normally distributed or as median (percentile $25-75$ ) if skewed. Categorical variables are expressed as frequencies and proportions (\%).

The one-way analysis of variance "ANOVA test" was used to compare the renal function estimates obtained with the MDRD4, CKD-EPI, and CG-BSA formulas.

Univariable time-to-event comparisons were performed using the log-rank test and survival was estimated with 
the Kaplan-Meier method. Cox proportional hazard regression models were used to model long-term survival as a function of the formulas both in univariable and multivariable analysis. Cox models assumptions were verified and $\mathrm{GFR} / \mathrm{CrCl}$ formulas were converted to restricted cubic splines to overcome linearity issues. An interaction term between the variable of interest and time was tested within the Cox model. In the multivariable models, the covariates were chosen from demographic (age and sex) and clinical (smoking, hypertension, diabetes, heart rate, and systolic blood pressure) parameters that were previously found to be clinically relevant and previously reported [42]. A significant "interaction" between age and "renal function" formulas was present in various populations; thus, these results are presented for age subgroups for which the "age" variable was not included in the adjustment models in order to decrease model instability. Left ventricular ejection fraction and hemoglobin were also not included in the "adjusted" models due to a high (>7 5\%) percentage of missing values.

Correlation estimates were verified prior to modeling and correlation coefficients $<0.6$ were considered to rule out noteworthy multicollinearity within the survival models [43].

To assess the relative importance and discriminative value of renal function estimators in terms of outcome, Harrell's c-index [44] was evaluated and compared with the correlated c-indices using the approach proposed by Kang et al. [45].

Calibration was assessed visually by plotting the mean of model-predicted survival at 2 years in each decile of predicted survival against the observed survival estimated by the Kaplan-Meier method. The higher discriminative value associated with the "net reclassification improvement" (NRI) was assessed at 2 years [46, 47]. This method assesses the ability of a new model to reclassify subjects with and without a clinical event during follow-up. The ability of the new model to reclassify is summarized by the NRI statistic. As previously used by our team [48], the continuous NRI method developed by Uno [47] and implemented in the survIDINRI package of the R software (The R Foundation for Statistical Computing) was used. The continuous NRI method does not require a prior definition of strata risk, thus considering the change in the estimation prediction as a continuous variable. The integrated discrimination improvement (IDI) for each eGFR and $\mathrm{CrCl}$ estimator was also calculated. The IDI evaluates the difference between the integrated sensitivity gain and the integrated specificity loss due to the addition of the eGFR and $\mathrm{CrCl}$ estimator to the prognostic model.

Statistical analyses were performed using SPSS 23 software (IBM Corp. Released 2013. IBM SPSS Statistics for Windows, Version 23.0. Armonk, NY: IBM Corp.) and the $\mathrm{R}$ software (The $\mathrm{R}$ Foundation for Statistical Computing).

A $P$ value of less than 0.05 was considered statistically significant.

\section{Results \\ Population characteristics and renal function stage classification}

A total of 2644 patients were analyzed in populationbased cohorts, 20,895 in CV risk cohorts, 1801 in HF populations, and 28,771 in post-MI cohorts.

The mean \pm SD age was $66.4 \pm 11.5,66.7 \pm 9.4,73.7 \pm$ 10.4 , and $65 \pm 11.5$ years in the population-based, $C V$ risk, HF, and post-MI populations, respectively. As expected, population-based cohorts had higher eGFR/CrCl while $\mathrm{HF}$ populations had lower eGFR/CrCl (as calculated by all formulas). Concordantly, the proportion of CVM was also lower in population-based cohorts while increasing progressively in $\mathrm{CV}$ risk, post-MI populations, and HF cohorts $(2.0 \%$ vs. $3.8 \%$ vs. $15.3 \%$ vs. $15.7 \%$, respectively; $P<0.001)$. The baseline characteristics of all populations are summarized in Table 1.

The CG-BSA formula reclassified a higher proportion of patients as having worse renal function ("stages $\geq 3$ ") in all populations. On the other hand, the proportion of CVM events (relative to the number of patients) was higher overall using the MDRD4 formula. For example, in population-based cohorts, patients reclassified into worse renal function stages experienced $6.9 \%$ of events according to the CG-BSA formula, $6.5 \%$ of events with CKD-EPI, and $7.2 \%$ of events with MDRD4 (similar observations were found in the other cohorts) (Table 2).

\section{Mortality prediction and accuracy}

The three formulas were effective with regard to CVM prediction. However, important differences were notably observed between populations and formulas.

Associations between the various "renal function" formulas and CVM within the studied populations are demonstrated in Table 3 for categorical variables and in Fig. 1 for "cubic spline transformed" continuous variables (linearity tests are shown in Additional file 1: Table S1). As continuous variables, all formulas showed to be independently associated with CVM in the various cohorts. An eGFR/CrCl lower than $60 \mathrm{~mL} / \mathrm{min} / 1.73 \mathrm{~m}^{2}$ was associated with an increase in event rate in all cohorts; however, the less accurate (wide confidence intervals) associations in population-based cohorts reflect the low CVM event rate in this setting (Fig. 1 and Additional file 1: Figure S1 for "log-transformed" hazard ratios). In population-based cohorts, the "categorical" MDRD4 formula lost its predictive value after adjustment for clinically relevant confounders (sex, smoking, 
Table 1 Baseline characteristics of the different cohorts

\begin{tabular}{|c|c|c|c|c|c|}
\hline Variables & Population-based & Cardiovascular risk & Heart failure & Post-myocardial infarction & $P$ value \\
\hline Number of patients & 2644 & 20,895 & 1801 & 28,771 & $<0.001$ \\
\hline \multicolumn{6}{|l|}{ Demographic } \\
\hline Age, years & $66.4 \pm 14.1$ & $66.7 \pm 9.4$ & $73.7 \pm 10.4$ & $65 \pm 11.5$ & $<0.001$ \\
\hline Male sex, n (\%) & $1359(51.4)$ & $14,083(67.4)$ & $1093(60.7)$ & $20,189(70.2)$ & $<0.001$ \\
\hline Smokers, n (\%) & $400(15.1)$ & $5994(28.8)$ & $185(10.7)$ & $18,235(63.4)$ & $<0.001$ \\
\hline \multicolumn{6}{|l|}{ Clinical and echocardiographic } \\
\hline Hypertension, n (\%) & $1397(53.1)$ & $17,892(85.6)$ & $861(47.8)$ & $15,570(54.1)$ & $<0.001$ \\
\hline Diabetes, n (\%) & $334(12.7)$ & $5164(24.7)$ & $541(30.1)$ & $7386(25.7)$ & $<0.001$ \\
\hline $\mathrm{SBP}, \mathrm{mmHg}$ & $136 \pm 18$ & $159 \pm 21$ & $133 \pm 24$ & $122 \pm 17$ & $<0.001$ \\
\hline Heart rate, bpm & $67 \pm 12$ & $71 \pm 13$ & $75 \pm 15$ & $76 \pm 13$ & $<0.001$ \\
\hline LVEF, $\%^{\mathrm{a}}$ & $67 \pm 8$ & $56 \pm 13$ & $41 \pm 15$ & $34 \pm 9$ & $<0.001$ \\
\hline Height, m & $1.67 \pm 8.9$ & $1.69 \pm 9.5$ & $1.66 \pm 9.7$ & $1.69 \pm 9.4$ & $<0.001$ \\
\hline Weight, kg & $73.8 \pm 13.9$ & $80.7 \pm 15.8$ & $76.4 \pm 18.2$ & $79.1 \pm 15.5$ & $<0.001$ \\
\hline \multicolumn{6}{|l|}{ Laboratory } \\
\hline Hemoglobin, $\mathrm{g} / \mathrm{dL}^{\mathrm{a}}$ & $13.9 \pm 1.3$ & $13.4 \pm 1.9$ & $13.2 \pm 1.8$ & $13.3 \pm 1.7$ & $<0.001$ \\
\hline Creatinine, mg/dL & $0.96 \pm 0.24$ & $1.12 \pm 0.25$ & $1.24 \pm 0.53$ & $1.12 \pm 0.32$ & $<0.001$ \\
\hline MDRD4, mL/min/1.73 $\mathrm{m}^{2}$ & $77.1 \pm 18.2$ & $66.8 \pm 15.3$ & $63.3 \pm 24.4$ & $71.05 \pm 39.61$ & $<0.001$ \\
\hline CKD-EPI, mL/min/1.73 m² & $74.6 \pm 17.0$ & $65.2 \pm 15.4$ & $59.3 \pm 21.9$ & $67.93 \pm 20.75$ & $<0.001$ \\
\hline CG-BSA, mL/min/1.73 $\mathrm{m}^{2}$ & $71.1 \pm 20.6$ & $64.6 \pm 17.8$ & $57.2 \pm 25.5$ & $68.32 \pm 32.89$ & $<0.001$ \\
\hline \multicolumn{6}{|l|}{ Follow-up and events } \\
\hline Follow-up, years & $4.2(3.6-4.9)$ & $5.0(3.3-5.9)$ & $2.6(1.2-3.5)$ & $1.0(0.2-1.9)$ & $<0.001$ \\
\hline CVM, n (\%) & $52(2.0)$ & 785 (3.8) & $283(15.7)$ & $4400(15.3)$ & $<0.001$ \\
\hline
\end{tabular}

$N$ number, $M I$ myocardial infarction, $M V$ missing values, SBP systolic blood pressure, LVEF left ventricular ejection fraction, CVM cardiovascular mortality, CG-BSA Cockcroft-Gault formula adjusted for body surface area, MDRD4 modification of diet in renal disease-4 formula, CKDEPI Chronic Kidney Disease Epidemiology Collaboration equation

Normally distributed variables are presented as mean \pm standard deviation; skewed variables are presented as median (percentile 25-75); proportions are presented as absolute numbers ( $\mathrm{n}$ ) and proportions (\%). $P$ values for "between group differences" were determined by one-way analysis of variance (ANOVA) tests ${ }^{a}$ These variables present $>75 \%$ missing values in all populations, therefore they are not included in the adjusted models

hypertension, diabetes, systolic blood pressure, and heart rate), whereas the "categorical" CKD-EPI and CG-BSA formulas remained significant for the lower renal function stages as compared to stage 1 (reference category) [HR for CKD-EPI stages 3b/4/5 = $6.43(95 \% \mathrm{CI}, 1.61-$ 25.65), $P=0.008$ and $\mathrm{HR}$ for CG-BSA stages $3 \mathrm{~b} / 4 / 5=$ 5.35 (95\% CI, 1.66-17.17), $P=0.005]$. In CV risk, HF, and post-MI populations, all "categorical" formulas were found to be independently associated with CVM for the lower "renal function" stages with intersecting confidence intervals (Table 3 ).

The associations of $\mathrm{GFR} / \mathrm{CrCl}$ formulas with cardiovascular mortality are mostly non-linear. In concordance, these results are presented as restricted cubic splines in Fig. 1. Interactions, linearity and colinearity were verified and excluded at each model step.

Long-term survival Kaplan-Meier curves are shown in Fig. 2. All formulas showed highly significant predictive prognostic values (log-rank test, $P<0.001$ ). However, eGFR/CrCl stages diverged in a more pronounced manner with the CG-BSA formula in all populations (higher $X^{2}$ values) (Fig. 2).

Significant "interactions" were frequently observed between age and the eGFR/CrCl formulas, suggesting that renal function is a more relevant prognosticator in younger populations (Additional file 1: Table S2).

\section{Discrimination, calibration, and reclassification improvement analysis}

In the CV risk, HF, and post-MI cohorts, the CG-BSA formula demonstrated statistically superior discriminative capacity (c-statistics) when added on top of a prognostic model (including gender, smoking, hypertension, diabetes status, heart rate and systolic blood pressure) as well as when compared to the GFR formulas (on top of the same model). Despite being statistical significant, the discriminative improvement driven by the CG-BSA formula was globally low (vs. eGFR formulas) ranging from 0.5 to $2 \%$ (Table 4 ). In population-based cohorts, the discriminative capacity improvement was not statistically 
Table 2 Proportion of patients within "renal function" categories according to the different formulas

\begin{tabular}{|c|c|c|c|c|c|c|c|}
\hline \multirow{2}{*}{$\begin{array}{l}\text { Formula } \\
\text { RF stage }\end{array}$} & \multicolumn{2}{|l|}{ MDRD4 } & \multicolumn{2}{|l|}{ CKD-EPI } & \multicolumn{2}{|l|}{ CG-BSA } & \multirow[t]{2}{*}{$P$ value } \\
\hline & Patients, n (\%) & Events, n (\%) & Patients, n (\%) & Events, n (\%) & Patients, n (\%) & Events, n (\%) & \\
\hline \multicolumn{8}{|l|}{ Population-based } \\
\hline Stage $1: \geq 90$ & $521(19.7)$ & $9(1.7)$ & $425(16.1)$ & $3(0.7)$ & $429(16.2)$ & $4(0.9)$ & $<0.001$ \\
\hline Stage 2: 60-89 & $1688(63.8)$ & $26(1.5)$ & $1701(64.3)$ & $29(1.7)$ & $1410(53.3)$ & $17(1.2)$ & $<0.001$ \\
\hline Stage 3a: 45-59 & $353(13.3)$ & $11(3.1)$ & $395(14.9)$ & $12(3.0)$ & $602(22.8)$ & $17(2.8)$ & $<0.001$ \\
\hline Stage $3 b / 4 / 5:<45$ & $83(3.1)$ & $6(7.2)$ & $123(4.7)$ & $8(6.5)$ & $203(7.7)$ & $14(6.9)$ & $<0.001$ \\
\hline \multicolumn{8}{|l|}{ Cardiovascular risk } \\
\hline Stage $1: \geq 90$ & $1223(5.9)$ & $27(2.2)$ & $1165(5.6)$ & $20(1.7)$ & $1727(8.3)$ & $28(1.6)$ & $<0.001$ \\
\hline Stage 2: 60-89 & $12,992(62.2)$ & $388(3.0)$ & $11,956(57.2)$ & $335(2.8)$ & $10,284(49.2)$ & $253(2.4)$ & $<0.001$ \\
\hline Stage 3a: 45-59 & $5325(25.5)$ & $242(4.5)$ & $5845(28.0)$ & $261(4.5)$ & $6277(30.0)$ & $293(4.7)$ & $<0.001$ \\
\hline Stage $3 b / 4 / 5:<45$ & $1355(6.5)$ & $128(9.4)$ & $1929(9.2)$ & $169(8.8)$ & $2607(12.5)$ & $211(8.1)$ & $<0.001$ \\
\hline \multicolumn{8}{|l|}{ Heart failure } \\
\hline Stage $1: \geq 90$ & $229(12.7)$ & $18(7.8)$ & $165(9.2)$ & $7(4.2)$ & $179(9.9)$ & $4(2.2)$ & $<0.001$ \\
\hline Stage 2: 60-89 & $738(41.0)$ & $77(10.4)$ & $685(38.0)$ & $70(10.2)$ & $533(29.6)$ & $45(8.4)$ & $<0.001$ \\
\hline Stage 3a: 45-59 & $405(22.5)$ & $71(17.5)$ & $428(23.8)$ & $63(14.7)$ & $443(24.6)$ & $62(14.0)$ & $<0.001$ \\
\hline Stage $3 b / 4 / 5:<45$ & $429(23.8)$ & $117(27.3)$ & $523(29.0)$ & $143(27.3)$ & $646(35.9)$ & $172(26.6)$ & $<0.001$ \\
\hline \multicolumn{8}{|c|}{ Post-myocardial infarction } \\
\hline Stage $1: \geq 90$ & $4277(15.3)$ & $405(9.5)$ & $4210(15.1)$ & $351(8.3)$ & $4315(15.8)$ & $337(7.8)$ & $<0.001$ \\
\hline Stage 2: 60-89 & $14,328(51.4)$ & $1670(11.7)$ & $13,400(48.1)$ & $1515(11.3)$ & $11,746(43.1)$ & $1197(10.2)$ & $<0.001$ \\
\hline Stage 3a: 45-59 & $6261(22.5)$ & $1247(19.9)$ & $6453(23.1)$ & $1228(19.0)$ & $6746(24.8)$ & $1219(18.1)$ & $<0.001$ \\
\hline Stage 3b: 30-45 & $2622(9.4)$ & $793(30.2)$ & $3174(11.4)$ & $924(29.1)$ & $3744(13.7)$ & $1090(29.1)$ & $<0.001$ \\
\hline Stage $4 / 5:<30$ & $388(1.5)$ & $162(41.8)$ & $639(2.4)$ & $259(40.5)$ & $682(2.6)$ & $280(41.1)$ & $<0.001$ \\
\hline
\end{tabular}

$R F$ renal function, CG-BSA Cockcroft-Gault formula adjusted for body surface area, MDRD4 modification of diet in renal disease-4 formula, CKD-EPI Chronic Kidney Disease Epidemiology Collaboration equation

Events refer to cardiovascular mortality

significant, nor superior to the eGFR formulas $(P \geq 0.05$ for all comparisons). The discriminative capacity of the CKD-EPI formula was superior to that of the MDRD4 formula in CV risk, HF, and post-MI cohorts, but not in population-based cohorts (Table 4).

The prognostic models were well calibrated, with intersecting predicted risks and confidence intervals of observed risks in all populations and formulas (Additional file 1: Figure S2). The IDI and NRI were higher $(P<0.05)$ for the CG-BSA formula as compared to MDRD4 and CKD-EPI in CV risk, HF, and post-MI cohorts. In the population-based cohorts, CG-BSA was not superior to the other formulas. The CKD-EPI formula was globally superior to MDRD4 (Table 4).

\section{Discussion}

\section{General interpretation}

The present study showed that CG-BSA was slightly more precise and accurate in terms of CVM prediction in $\mathrm{CV}$ risk, HF, and post-MI cohorts, but not in population-based cohorts, followed by the CKD-EPI formula. The CG-BSA formula estimates $\mathrm{CrCl}$ (and not the
GFR contrary to the MDRD4 and CKD-EPI formulas) and requires individual height and weight for its computation. Therefore, CKD-EPI offers the best compromise between renal function estimation and CVM prediction.

\section{Development and validation of $\mathrm{GFR}$ and $\mathrm{CrCl}$ formulas}

Estimation of GFR (and $\mathrm{CrCl}$ in the case of CG/CGBSA formulas) is an inexpensive, practical, and fairly reliable means to assess renal function in clinical practice [5]. However, certain drawbacks need to be considered since creatinine-based eGFR/CrCl is dependent on multiple factors including age, sex, race, diet, muscle mass, tubular secretion, unstable renal function, and BSA [16]. Hence, the varying performance of the equations is likely to rely on their "core formula" development and validation.

The equation proposed by Cockcroft and Gault in 1976 was developed in a Caucasian male population of 236 patients aged $18-92$ years in order to predict $\mathrm{CrCl}$ (and not GFR) in situations in which renal function was only slightly impaired [11]. This original CG formula was found to be inaccurate for GFR prediction. The CG 
Table 3 Cox-regression models for cardiovascular mortality according to the different formulas and cohorts

\begin{tabular}{|c|c|c|c|c|}
\hline Variables & Univariable: HR (95\% Cl) & $P$ value & Adjusted: HR (95\% Cl) & $P$ value \\
\hline \multicolumn{5}{|l|}{ Population-based } \\
\hline \multicolumn{5}{|l|}{ MDRD4 } \\
\hline Categorical & & 0.001 & & 0.046 \\
\hline Stage $1: \geq 90$ & Reference & & Reference & \\
\hline Stage 2: 60-89 & $0.818(0.382-1.751)$ & 0.605 & $0.799(0.370-1.726)$ & 0.568 \\
\hline Stage 3a: 45-59 & 1.908 (0.790-4.609) & 0.151 & $1.423(0.563-3.597)$ & 0.456 \\
\hline Stage $3 b / 4 / 5:<45$ & $4.624(1.644-13.008)$ & 0.004 & $3.004(0.976-9.247)$ & 0.055 \\
\hline \multicolumn{5}{|l|}{ CKD-EPI } \\
\hline Categorical & & $<0.001$ & & 0.019 \\
\hline Stage $1: \geq 90$ & Reference & & Reference & \\
\hline Stage 2: 60-89 & $2.471(0.752-8.117)$ & 0.136 & $2.007(0.607-6.640)$ & 0.254 \\
\hline Stage 3a: 45-59 & $4.968(1.400-17.635)$ & 0.013 & $3.122(0.851-11.456)$ & 0.086 \\
\hline Stage 3b/4/5: < 45 & 11.207 (2.967-42.335) & $<0.001$ & $6.426(1.610-25.652)$ & 0.008 \\
\hline \multicolumn{5}{|l|}{ CG-BSA } \\
\hline Categorical & & $<0.001$ & & $<0.001$ \\
\hline Stage $1: \geq 90$ & Reference & & Reference & \\
\hline Stage 2: 60-89 & $1.367(0.459-4.070)$ & 0.574 & $1.078(0.360-3.230)$ & 0.894 \\
\hline Stage 3a: 45-59 & $3.658(1.226-10.915)$ & 0.020 & $2.393(0.787-7.275)$ & 0.124 \\
\hline Stage 3b/4/5: < 45 & $9.562(3.131-29.199)$ & $<0.001$ & $5.347(1.665-17.170)$ & 0.005 \\
\hline \multicolumn{5}{|l|}{ Cardiovascular risk } \\
\hline \multicolumn{5}{|l|}{ MDRD4 } \\
\hline Categorical & & $<0.001$ & & $<0.001$ \\
\hline Stage $1: \geq 90$ & Reference & & Reference & \\
\hline Stage 2: 60-89 & $1.008(0.682-1.489)$ & 0.969 & $1.096(0.740-1.624)$ & 0.647 \\
\hline Stage 3a: 45-59 & $1.682(1.130-2.504)$ & 0.010 & $1.924(1.285-2.881)$ & 0.001 \\
\hline Stage $3 b / 4 / 5:<45$ & 4.308 (2.844-6.526) & $<0.001$ & $5.138(3.361-7.857)$ & $<0.001$ \\
\hline \multicolumn{5}{|l|}{ CKD-EPI } \\
\hline Categorical & & $<0.001$ & & $<0.001$ \\
\hline Stage 1: $\geq 90$ & Reference & & Reference & \\
\hline Stage 2: 60-89 & $1.292(0.823-2.029)$ & 0.266 & $1.391(0.884-2.190)$ & 0.154 \\
\hline Stage 3a: 45-59 & $2.263(1.436-3.566)$ & $<0.001$ & $2.543(1.605-4.029)$ & $<0.001$ \\
\hline Stage $3 b / 4 / 5:<45$ & $5.397(3.393-8.583)$ & $<0.001$ & $6.292(3.923-10.091)$ & $<0.001$ \\
\hline \multicolumn{5}{|l|}{ CG-BSA } \\
\hline Categorical & & $<0.001$ & & $<0.001$ \\
\hline Stage $1: \geq 90$ & Reference & & Reference & \\
\hline Stage 2: 60-89 & $1.347(0.911-1.990)$ & 0.135 & $1.504(1.009-2.241)$ & 0.045 \\
\hline Stage 3a: 45-59 & $2.927(1.986-4.315)$ & $<0.001$ & $3.416(2.290-5.094)$ & $<0.001$ \\
\hline Stage $3 b / 4 / 5:<45$ & $6.277(4.227-9.322)$ & $<0.001$ & $7.520(4.981-11.353)$ & $<0.001$ \\
\hline \multicolumn{5}{|l|}{ Heart failure } \\
\hline \multicolumn{5}{|l|}{ MDRD4 } \\
\hline Categorical & & $<0.001$ & & $<0.001$ \\
\hline Stage $1: \geq 90$ & Reference & & Reference & \\
\hline Stage 2: $60-89$ & $1.076(0.643-1.800)$ & 0.781 & $1.016(0.605-1.706)$ & 0.952 \\
\hline Stage 3a: 45-59 & $1.791(1.066-3.007)$ & 0.028 & $1.517(0.896-2.569)$ & 0.121 \\
\hline Stage $3 b / 4 / 5:<45$ & $3.298(2.007-5.420)$ & $<0.001$ & $2.824(1.704-4.680)$ & $<0.001$ \\
\hline
\end{tabular}


Table 3 Cox-regression models for cardiovascular mortality according to the different formulas and cohorts (Continued)

\begin{tabular}{|c|c|c|c|c|}
\hline \multicolumn{5}{|l|}{ CKD-EPI } \\
\hline Categorical & & $<0.001$ & & $<0.001$ \\
\hline Stage $1: \geq 90$ & Reference & & Reference & \\
\hline Stage 2: 60-89 & $2.194(1.008-4.774)$ & 0.048 & $2.248(1.030-4.906)$ & 0.042 \\
\hline Stage 3a: 45-59 & 2.997 (1.372-6.549) & 0.006 & $2.871(1.306-6.310)$ & 0.009 \\
\hline Stage $3 b / 4 / 5:<45$ & $6.557(3.070-14.006)$ & $<0.001$ & $5.784(2.692-12.430)$ & $<0.001$ \\
\hline \multicolumn{5}{|l|}{ CG-BSA } \\
\hline Categorical & & $<0.001$ & & $<0.001$ \\
\hline Stage $1: \geq 90$ & Reference & & Reference & \\
\hline Stage 2: 60-89 & $3.243(1.164-9.031)$ & 0.024 & $3.457(1.240-9.642)$ & 0.018 \\
\hline Stage 3a: 45-59 & $5.266(1.915-14.485)$ & 0.001 & $5.078(1.838-14.024)$ & 0.002 \\
\hline Stage $3 b / 4 / 5:<45$ & $12.388(4.595-33.398)$ & $<0.001$ & 11.006 (4.066-29.793) & $<0.001$ \\
\hline \multicolumn{5}{|l|}{ Post-myocardial infarction } \\
\hline \multicolumn{5}{|l|}{ MDRD4 } \\
\hline Categorical & & $<0.001$ & & $<0.001$ \\
\hline Stage 1: $\geq 90$ & Reference & & Reference & \\
\hline Stage 2: 60-89 & $1.243(1.115-1.385)$ & $<0.001$ & $1.296(1.162-1.446)$ & $<0.001$ \\
\hline Stage 3a: 45-59 & $2.253(2.014-2.521)$ & $<0.001$ & 2.251 (2.009-2.523) & $<0.001$ \\
\hline Stage 3b: $30-45$ & $3.692(3.276-4.162)$ & $<0.001$ & 3.509 (3.104-3.967) & $<0.001$ \\
\hline Stage $4 / 5:<30$ & $5.509(4.591-6.610)$ & $<0.001$ & $5.076(4.208-6.123)$ & $<0.001$ \\
\hline \multicolumn{5}{|l|}{ CKD-EPI } \\
\hline Categorical & & $<0.001$ & & $<0.001$ \\
\hline Stage $1: \geq 90$ & Reference & & Reference & \\
\hline Stage 2: 60-89 & $1.363(1.214-1.531)$ & $<0.001$ & $1.427(1.269-1.603)$ & $<0.001$ \\
\hline Stage 3a: 45-59 & $2.430(2.158-2.736)$ & $<0.001$ & $2.442(2.165-2.754)$ & $<0.001$ \\
\hline Stage 3b: $30-45$ & $3.976(3.516-4.497)$ & $<0.001$ & $3.852(3.396-4.368)$ & $<0.001$ \\
\hline Stage $4 / 5:<30$ & $6.098(5.193-7.160)$ & $<0.001$ & $5.765(4.887-6.800)$ & $<0.001$ \\
\hline \multicolumn{5}{|l|}{ CG-BSA } \\
\hline Categorical & & $<0.001$ & & $<0.001$ \\
\hline Stage $1: \geq 90$ & Reference & & Reference & \\
\hline Stage 2: 60-89 & $1.302(1.154-1.470)$ & $<0.001$ & $1.369(1.213-1.546)$ & $<0.001$ \\
\hline Stage 3a: 45-59 & $2.447(2.169-2.761)$ & $<0.001$ & $2.523(2,234-2.849)$ & $<0.001$ \\
\hline Stage 3b: 30-45 & $4.230(3.744-4.780)$ & $<0.001$ & 4.239 (3.745-4.799) & $<0.001$ \\
\hline Stage 4/5: < 30 & $6.536(5.578-7.659)$ & $<0.001$ & 6.639 (5.649-7.802) & $<0.001$ \\
\hline
\end{tabular}

formula incorporates glomerular $\mathrm{CrCl}$ plus tubular $\mathrm{CrCl}$, resulting in an overestimation of GFR in younger healthy populations [12, 13], whereas in older populations, it may underestimate GFR due to its formula computation, i.e., the numerator includes "140-age"; thus, for the same weight and creatinine, a very old patient will have a disproportionate underestimation of their GFR [14, 15]. Despite the fact that CG adjusted for BSA may improve its accuracy $[16,49,50]$, it should be emphasized that neither the original CG nor the BSA-adjusted formula should be used for GFR estimation and that these formulas are not recommended by updated guidelines. Still, many clinicians and laboratories continue to use the latter on a daily basis [10]. In the late nineties, the MDRD group developed models that improved the prediction of eGFR from plasma creatinine concentration [6], providing more reliable estimations of kidney function than the CG-BSA formula [51]. Finally, the 


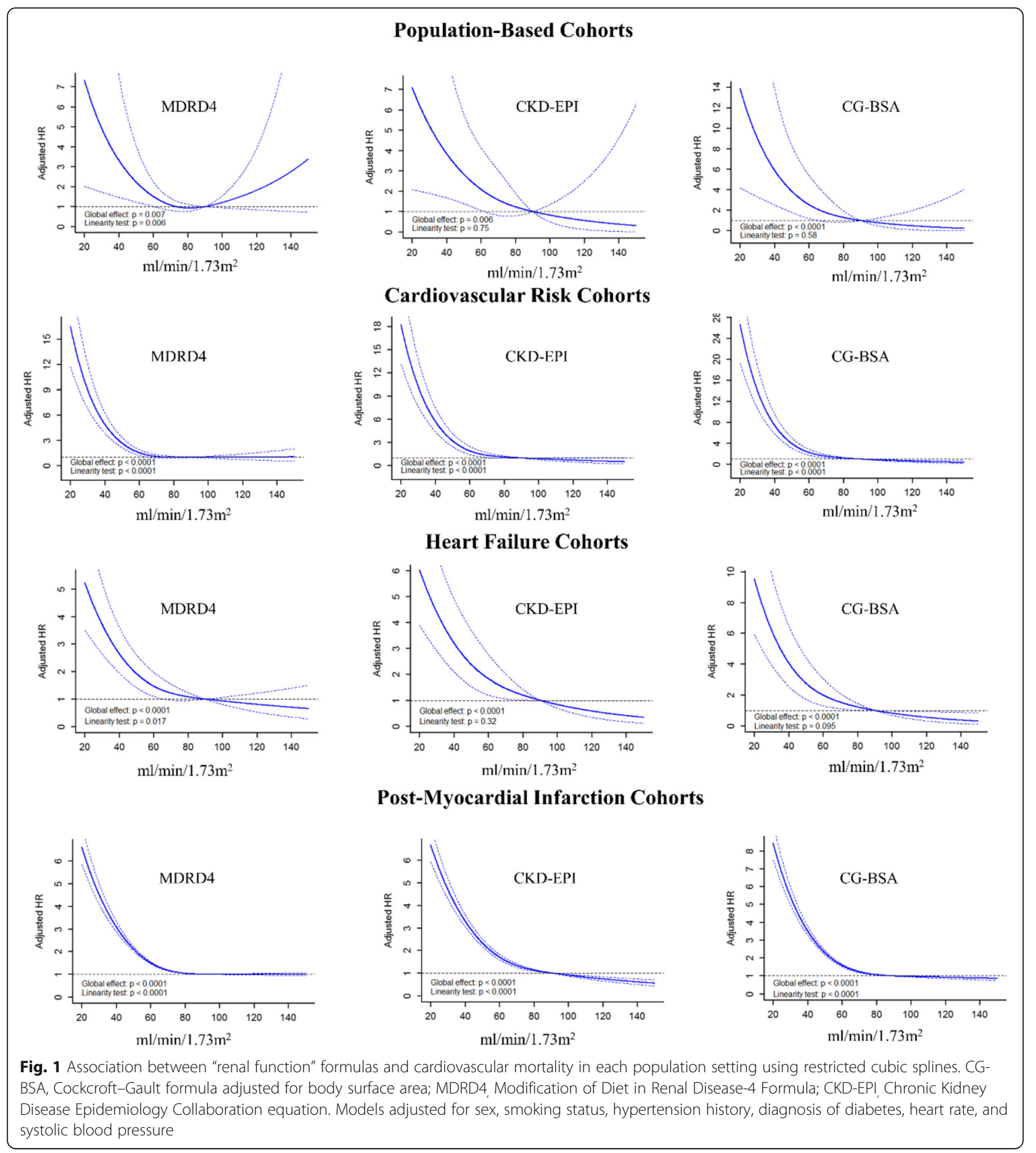

CKD-EPI equation was developed from a population of more than 16,000 participants. This formula was validated and found to be more accurate than the MDRD4 formula (compared to a renal-clearance "gold standard"), and thus proposed as a first choice to estimate GFR in routine clinical practice $[4,9]$.
It should also be noted that the MDRD and CKDEPI equations allow GFR estimation without the need of available "individualized" weight or height (as most laboratories do not assess these data), this information being standardized in the formulas' "intrinsic design" [52]. 


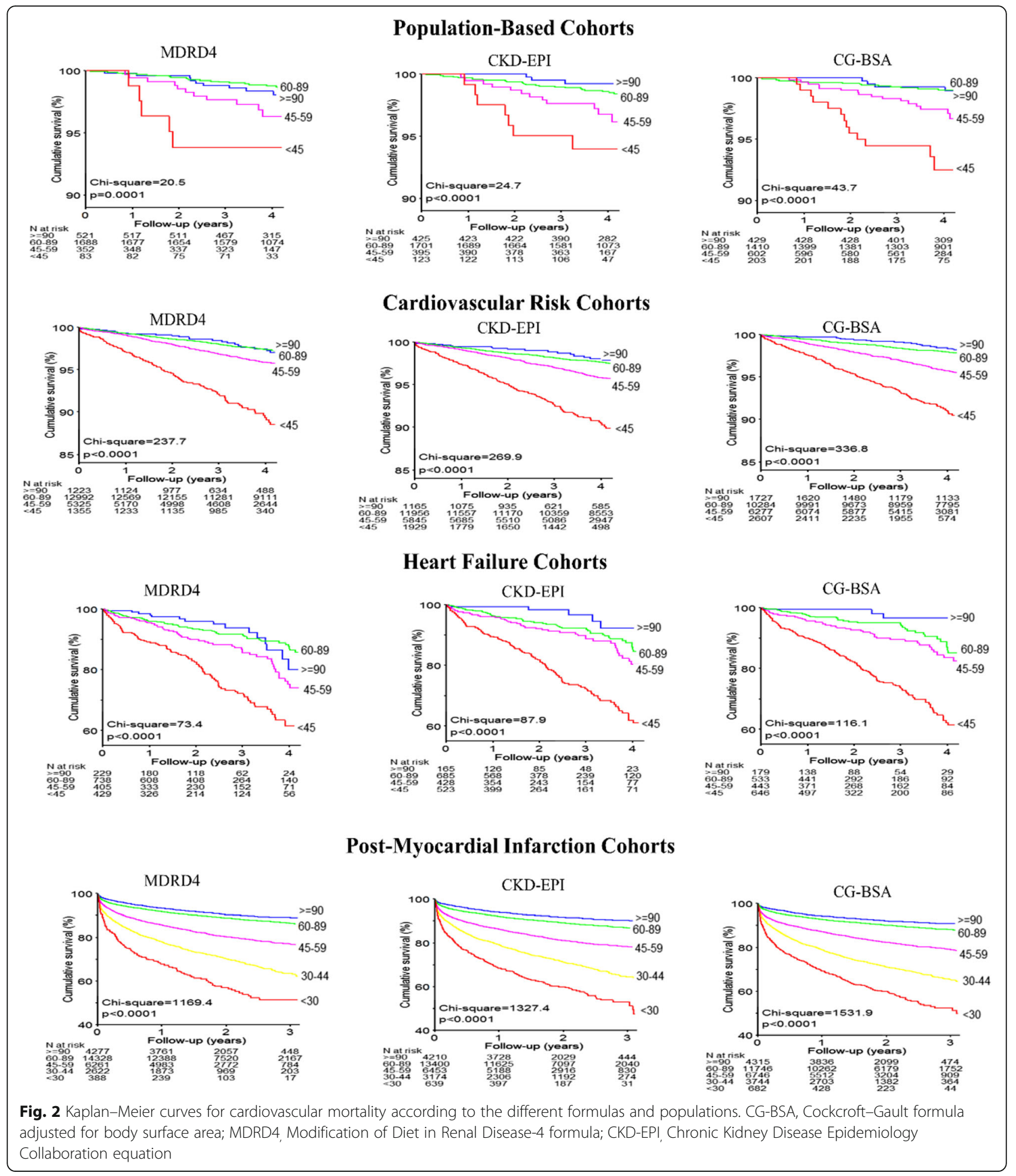

Comparing eGFR/CrCl formulas to a renal-clearance "gold-standard" in large populations clearly underscores the lack of precision of all these formulas [51, 52]. Additionally, the eGFR/ $\mathrm{CrCl}$ formulas are prone to high misclassification rates $(\geq 30 \%)$ of patients according to the Kidney Disease Outcomes Quality Initiative Chronic Kidney Disease (K/DOQI-CKD) classification stages [51-53].

Still, the CKD-EPI formula outperforms the MDRD4 and CG/CG-BSA in terms of eGFR precision and 
Table 4 C-statistics, integrated discrimination improvement, and net reclassification improvement of restricted cubic spline "renal function" formulas for cardiovascular mortality discrimination and reclassification in the studied cohorts

\begin{tabular}{|c|c|c|c|c|c|c|c|c|c|}
\hline Cohorts & $\begin{array}{l}\text { Model comparison } \\
\text { (A vs. B) }\end{array}$ & C-index A\% & C-index B\% & $\Delta \mathrm{C}$-index\% & $P$ value & $\mathrm{ID} / \%(95 \% \mathrm{Cl})$ & $P$ value & NRI\% (95\% Cl) & $P$ value \\
\hline \multirow[t]{6}{*}{ Population } & Model vs. MDRD4 & 72.8 & 75.7 & $2.9(-0.3$ to 6.1$)$ & 0.072 & $0.5(-0.1$ to 4.3$)$ & 0.16 & $16.7(-2.0$ to 44.1$)$ & 0.072 \\
\hline & Model vs. CKD-EPI & 72.8 & 75.2 & $2.4(-1.6$ to 6.4$)$ & 0.23 & 0.6 (0.1 to 4.8$)$ & 0.016 & $23.4(-0.9$ to 44.4$)$ & 0.054 \\
\hline & Model vs. CG-BSA & 72.8 & 77.3 & 4.5 (-0.7 to 9.7$)$ & 0.087 & 1.0 (0.2 to 5.7$)$ & 0.020 & 39.1 (4.6 to 51.2 ) & 0.020 \\
\hline & MDRD4 vs. CKD-EPI & 75.7 & 75.2 & $0.5(-2.4$ to 3.4$)$ & 0.74 & 0.2 (0.0 to 0.8 ) & 0.026 & $23.2(-10.7$ to 36.8$)$ & 0.20 \\
\hline & MDRD4 vs. CG-BSA & 75.7 & 77.3 & $1.6(-2.8$ to 6.1$)$ & 0.48 & 0.5 (0.0 to 2.1$)$ & 0.045 & $27.4(-6.8$ to 47.9$)$ & 0.13 \\
\hline & CKD-EPI vs. CG-BSA & 75.2 & 77.3 & $2.1(-0.1$ to 4.4$)$ & 0.067 & $0.4(-0.2$ to 1.6$)$ & 0.14 & $31.3(-13.9$ to 48.1$)$ & 0.17 \\
\hline \multirow[t]{6}{*}{ CV risk } & Model vs. MDRD4 & 58.9 & 66.2 & 7.3 (5.2 to 9.5$)$ & $<0.001$ & 0.9 (0.6 to 1.6$)$ & $<0.001$ & 18.5 (13.0 to 24.3$)$ & $<0.001$ \\
\hline & Model vs. CKD-EPI & 58.9 & 67.5 & 8.6 (6.4 to 10.9$)$ & $<0.001$ & 1.1 (0.7 to 1.7$)$ & $<0.001$ & 20.3 (15.3 to 25.8 ) & $<0.001$ \\
\hline & Model vs. CG-BSA & 58.9 & 70.2 & 11.3 (8.9 to 13.7 ) & $<0.001$ & 1.5 (1.0 to 2.4$)$ & $<0.001$ & 27.3 (21.4 to 32.6) & $<0.001$ \\
\hline & MDRD4 vs. CKD-EPI & 66.2 & 67.5 & 1.3 (0.8 to 1.8$)$ & $<0.001$ & 0.1 (0.1 to 0.2 ) & 0.006 & 23.0 (16.7 to 29.5$)$ & $<0.001$ \\
\hline & MDRD4 vs. CG-BSA & 66.2 & 70.2 & $4.0(2.7$ to 5.3$)$ & $<0.001$ & 0.6 (0.3 to 0.9$)$ & $<0.001$ & 22.8 (16.8 to 29.1$)$ & $<0.001$ \\
\hline & CKD-EPI vs. CG-BSA & 67.5 & 70.2 & 2.7 (1.7 to 3.7$)$ & $<0.001$ & $0.4(0.2$ to 0.7$)$ & $<0.001$ & 21.1 (14.6 to 27.6) & $<0.001$ \\
\hline \multirow[t]{6}{*}{ Heart failure } & Model vs. MDRD4 & 70.7 & 75.0 & 4.2 (1.9 to 6.5$)$ & $<0.001$ & 2.7 (1.2 to 4.8$)$ & $<0.001$ & 24.6 (15.9 to 34.1$)$ & $<0.001$ \\
\hline & Model vs. CKD-EPI & 70.7 & 75.5 & 4.7 (2.3 to 7.1$)$ & $<0.001$ & 3.0 (1.5 to 5.2$)$ & $<0.001$ & 25.5 (15.5 to 34.1$)$ & $<0.001$ \\
\hline & Model vs. CG-BSA & 70.7 & 77.0 & 6.3 (3.6 to 8.9) & $<0.001$ & 4.4 (2.6 to 7.2$)$ & $<0.001$ & 29.6 (20.1 to 39.9) & $<0.001$ \\
\hline & MDRD4 vs. CKD-EPI & 75.0 & 75.5 & 0.5 (0.2 to 0.8$)$ & 0.003 & 0.3 (0.1 to 0.5$)$ & 0.006 & 12.7 (1.6 to 23.8 ) & 0.024 \\
\hline & MDRD4 vs. CG-BSA & 75.0 & 77.0 & 2.1 (1.1 to 3.0$)$ & $<0.001$ & 1.7 (0.8 to 2.7 ) & $<0.001$ & 24.9 (14.7 to 34.7$)$ & $<0.001$ \\
\hline & CKD-EPI vs. CG-BSA & 75.5 & 77.0 & 1.6 (0.8 to 2.4$)$ & $<0.001$ & 1.4 (0.6 to 2.3 ) & $<0.001$ & 26.5 (14.4 to 34.5$)$ & $<0.001$ \\
\hline \multirow[t]{6}{*}{ Post-Ml } & Model vs. MDRD4 & 61.7 & 66.5 & 4.9 (4.2 to 5.6$)$ & $<0.001$ & 3.4 (2.9 to 3.9$)$ & $<0.001$ & 21.1 (19.4 to 22.8) & $<0.001$ \\
\hline & Model vs. CKD-EPI & 61.7 & 67.3 & 5.6 (4.9 to 6.4$)$ & $<0.001$ & 3.8 (3.4 to 4.4 ) & $<0.001$ & 22.4 (20.6 to 24.0$)$ & $<0.001$ \\
\hline & Model vs. CG-BSA & 61.7 & 68.8 & 7.1 (6.3 to 7.9$)$ & $<0.001$ & 4.9 (4.3 to 5.6$)$ & $<0.001$ & 24.9 (23.0 to 26.5$)$ & $<0.001$ \\
\hline & MDRD4 vs. CKD-EPI & 66.5 & 67.3 & 0.8 (0.6 to 0.9$)$ & $<0.001$ & 0.5 (0.4 to 0.6$)$ & $<0.001$ & 16.4 (13.6 to 20.0) & $<0.001$ \\
\hline & MDRD4 vs. CG-BSA & 66.6 & 68.8 & 2.3 (1.9 to 2.6$)$ & $<0.001$ & $1.6(1.3$ to 1.9$)$ & $<0.001$ & 20.9 (18.9 to 23.1) & $<0.001$ \\
\hline & CKD-EPI vs. CG-BSA & 67.3 & 68.8 & 1.5 (1.3 to 1.8$)$ & $<0.001$ & 1.1 (0.9 to 1.4$)$ & $<0.001$ & 16.6 (13.8 to 20.4) & $<0.001$ \\
\hline
\end{tabular}

CG-BSA Cockcroft-Gault formula adjusted for body surface area, MDRD4 modification of diet in renal disease-4 formula, CKD-EPI Chronic Kidney Disease Epidemiology Collaboration equation, $C V$ cardiovascular, $M I$ myocardial infarction, $I D I$ integrated discrimination improvement, $N R I$ net reclassification improvement, $\Delta$ delta (change) All models adjusted for gender, smoking status, history of hypertension, diagnosis of diabetes, heart rate and systolic blood pressure (not adjusted for hemoglobin or left ventricular ejection fraction due to $>75 \%$ of missing values in all datasets)

All "renal function" formulas are analyzed using restricted cubic splines

classification in several populations (compared to a "gold standard") [52], although this is not necessarily the case for outcomes prediction as discussed below.

\section{Estimation of GFR and $\mathrm{CrCl}$ versus mortality prediction}

GFR estimation serves not only to estimate renal function (for which purpose the CKD-EPI formula is the most accurate to date), but also to estimate the risk of major outcomes, such as cardiovascular mortality, since renal function is the strongest mortality predictor in many populations $[2,3,5]$. In this regard, the CKD-EPI formula was also found to be superior to MDRD4 for mortality prediction in large general population cohorts, high vascular risk cohorts, and chronic kidney disease cohorts [3]. Specifically, a meta-analysis by Matsushita et al. [3] evaluated the risk implications of the CKD-EPI formula as compared to the MDRD equation in populations comprising 1,130,472 adults from 25 general populations, 7 high-risk (of vascular disease), and 13 CKD cohorts. In this latter study, the CKD-EPI equation reclassified fewer individuals as CKD and more accurately categorized the risk for mortality and ESRD than did the MDRD equation. Nonetheless, the CG-BSA formula has not been consistently used for risk prediction purposes in these population settings, since it does not estimate GFR $[3,22,23,54]$, with the exception of a recent study comparing CG-BSA, CKD-EPI, and MDRD4 in 925 ambulatory HF patients where the CG-BSA formula also showed superiority for mortality risk prediction [19]. Our study distinguishes from previous reports by using the CG formula adjusted for BSA, and reveals a slight superiority of CG-BSA over CKD-EPI and MDRD4 formulas in predicting cardiovascular mortality in the studied CV risk, HF, and post-MI cohorts, but not 
in population-based cohorts. The CKD-EPI formula also showed good accuracy for CVM prediction and was globally superior to the MDRD4 formula. A possible explanation for the mild improvement in predictive value of the CG-BSA formula, as compared to the MDRD4 and CKD-EPI formulas, is the use of "individually observed" BSA in the CG-BSA formula computation versus "intrinsic design" BSA in the MDRD4 and CKD-EPI formulas, as "individual" BSA carries important prognostic information per se [55]. While the addition of BSA on top of CG-BSA did not improve reclassification indices in an exploratory analysis, these indices were nonetheless improved when BSA was added to MDRD4 and CKD-EPI, supporting that the "individual" BSA can provide prognostic information on top of the "intrinsic design" BSA of MDRD and CKD-EPI (Additional file 1: Table S3) $[52,55]$. Another potential explanation is that all of the studied cohorts herein consisted of elderly populations, in which both the CG and CG-BSA are likely to underestimate renal function, as previously highlighted, thereby "reclassifying" more patients into worse renal function categories (as demonstrated in Table 2). However, the CKD-EPI formula provides more accurate renal function estimations (as also discussed above) as well as good CVM prediction. Moreover, it does not require individual height or weight, making this formula easy to implement and favoring its widespread use. Despite the statistically superior discriminative capacity of CG-BSA (as compared to the other formulas) in $\mathrm{CV}$ risk, HF, and post-MI cohorts, it is unlikely to be clinically relevant, with CKD-EPI offering the best compromise between renal function estimation and CVM prediction/risk assessment.

\section{Innovation and clinical and research implications}

Globally, the greater accuracy of the CG-BSA equation for CVM prediction has the potential to slightly improve prognostic information in $\mathrm{CV}$ risk, HF, and post-MI populations [4]. However, the CG-BSA does not accurately estimate renal function and is only slightly superior statistically (and clinically irrelevant) comparatively to CKD-EPI, which remains the best formula overall.

\section{Limitations}

Several limitations of this study should be acknowledged. First, eGFR/CrCl was tested for cardiovascular mortality prediction and not for GFR accuracy, and therefore the best formula to estimate "actual" GFR cannot be derived from this study. Second, data regarding cystatin-C or microalbuminuria levels were unavailable; these data would have likely enhanced risk prediction models since non-GFR mechanisms (such as microalbuminuria) are also associated with prognosis and models incorporating both GFR and microalbuminuria perform more accurately in prognosis prediction [56]. Third, in a small number of patients, the fact that the reference weight at admission used for the CG formula may not have been the true dry weight and thus potentially overestimated cannot be disregarded. Fourth, creatinine was measured at baseline, whereas the study did not account for "time-dependent" variations that could also have major prognostic implications. Fifth, creatinine measurements were not "standardized" between different populations and cohorts; however, this potential heterogeneity in creatinine values was not systematic, reinforcing the strength of our results. Sixth, in the computation of CG-BSA, height and weight were added in an individual basis, it is thus necessary for these data to be available (limiting a wider applicability of this formula). Finally, the populations included in the studied cohorts all had a mean age above 65 years such that the CG formula (independently of BSA adjustment) is likely to underestimate renal function (as described in the discussion section) and thus reclassify a higher proportion of patients into lower renal function stages; consequently, these data may not be replicable in younger populations.

\section{Conclusion}

The CG-BSA formula was the most accurate in predicting CVM in CV risk, HF, and post-MI cohorts, but not in population-based cohorts. However, the CG-BSA discriminative improvement was globally low compared to MDRD4 and especially CKD-EPI formulas. In addition, CG-BSA is inaccurate for renal function estimation and has limited generalizability due to the need for individual height and weight values. Therefore, the CKD-EPI formula offers the best "global package" of renal function estimation and CVM prediction.

\section{Additional file}

Additional file 1: Supplemental Material. Table S1. Linearity tests for each formula using restricted cubic splines with 3 knots. Table S2. Cox-regression models according to the different formulas (categorized for "renal function" stages) and age subgroups. Table S3. Improvement indices for glomerular filtration rate estimation formulas in comparison to body surface area. Figure S1. Association between "renal function" formulas and cardiovascular mortality in each population setting using restricted cubic splines with y-axis in log scale. Figure S2. Calibration assessment for "renal function" formulas within each population. (DOCX $883 \mathrm{~kb})$

\section{Acknowledgments}

The authors acknowledge Pierre Pothier for the editing of the manuscript. 


\section{Authors' contributions}

JPF, NG, KD, FZ and PR made substantial contributions to conception, design, analysis and interpretation of data; they drafted the manuscript and gave final approval of the version to be published; they agreed to be accountable for all aspects of the work in ensuring that questions related to the accuracy or integrity of any part of the work are appropriately investigated and resolved. PP, SG, MAP, JJVM, BP, KD, LJ, JAS, JB, RL, SM, AM, HPBLR, CD, SH, NS, JWJ, and JGC provided relevant intellectual content, gave final approval of the version to be published and agreed to be accountable for all aspects of the work in ensuring that questions related to the accuracy or integrity of any part of the work are appropriately investigated and resolved. All authors read and approved the final manuscript.

\section{Competing interests}

The authors declare that they have no competing interests.

\section{Ethics approval and consent to participate}

Ethical approval was provided in accordance with the Declaration of Helsinki by the ethics committees of the respective projects (the "high-risk myocardial infarction initiative" and the "heart-omics in ageing").

\section{Author details}

'INSERM, Centre d'Investigations Cliniques Plurithématique 1433, INSERM U1116, Université de Lorraine, CHRU de Nancy, F-CRIN INI-CRCT, Nancy, France. ${ }^{2}$ Academic Cardiology Unit, University of Hull, Castle Hill Hospital, Kingston upon Hull, UK. ${ }^{3}$ Université de Lorraine, Institut Elie Cartan de Lorraine, UMR 7502, Vandoeuvre-lès-Nancy F-54506, France. ${ }^{4}$ CNRS, Institut Elie Cartan de Lorraine, UMR 7502, Vandoeuvre-lès-Nancy F-54506, France. ${ }^{5}$ Team BIGS, INRIA, Villers-lès-Nancy F-54600, France. ${ }^{6}$ Division of Cardiovascular Medicine, Brigham \& Women's Hospital, Harvard Medical School, Boston, Massachusetts 02115, USA. ${ }^{7}$ BHF Cardiovascular Research Centre, University of Glasgow, Glasgow, Scotland, UK. ${ }^{8}$ Department of Medicine, University of Michigan School of Medicine, Ann Arbor, MA, USA. ${ }^{9}$ ASH Comprehensive Hypertension Center, Division of Endocrinology, Diabetes, and Metabolism, Department of Medicine, The University of Chicago, Chicago, IL, USA. ${ }^{10}$ Department of Cardiology, University of Bergan, Stavanger University Hospital, Stavanger, Norway. ${ }^{11}$ Studies Coordinating Centre, Research Unit Hypertension and Cardiovascular Epidemiology, KU Leuven Department of Cardiovascular Sciences, University of Leuven, Leuven, Belgium. ${ }^{12}$ Cardiology Division, Stony Brook University, Stony Brook, NY, USA. ${ }^{13}$ Laboratory of Cardiovascular Clinical Pharmacology, IRCCS - Istituto di Ricerche Farmacologiche 'Mario Negri', Milan, Italy. ${ }^{14}$ Hôpital Lariboisière, Université Paris Diderot, Inserm 942, Paris, France. ${ }^{15}$ Cardiovascular Research Institute Maastricht, Maastricht University Medical Centre, Maastricht, The Netherlands Department of Cardiology, Maastricht University Medical Centre, Maastricht, The Netherlands. ${ }^{16}$ Department of Cardiology, Maastricht University Medical Center, Postbox 5800, 6202, AZ, Maastricht, The Netherlands. ${ }^{17}$ Department of Cardiology and Einthoven Laboratory for Experimental Vascular Medicine, Leiden University Medical Center, Leiden, The Netherlands. ${ }^{18}$ Interuniversity Cardiology Institute of the Netherlands, Utrecht, The Netherlands. ${ }^{19}$ National Heart and Lung Institute, Imperial College London (Royal Brompton and Harefield Hospitals) Department of Cardiology, Castle Hill Hospital, University of Hull, Hull, UK. ${ }^{20}$ Centre d'Investigations Cliniques-INSERM CHU de Nancy, Institut Lorrain du Cœur et des Vaisseaux Louis Mathieu, 4 Rue du Morvan, 54500 Vandoeuvre lès Nancy, France.

\section{Received: 30 June 2016 Accepted: 26 October 2016}

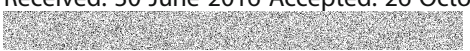

\section{References}

1. Anavekar NS, McMurray JJ, Velazquez EJ, et al. Relation between renal dysfunction and cardiovascular outcomes after myocardial infarction. N Engl J Med. 2004;351:1285-95.

2. Moukarbel GV, Yu ZF, Dickstein $K$, et al. The impact of kidney function on outcomes following high risk myocardial infarction: findings from 27610 patients. Eur J Heart Fail. 2014;16(3):289-99.

3. Matsushita K, Mahmoodi BK, Woodward M, et al. Comparison of risk prediction using the CKD-EPI equation and the MDRD study equation for estimated glomerular filtration rate. JAMA. 2012;307:1941-51.
4. Levey AS, Stevens LA, Schmid CH, et al. A new equation to estimate glomerular filtration rate. Ann Intern Med. 2009;150:604-12.

5. Levey AS, Coresh J, Balk E, et al. National Kidney Foundation practice guidelines for chronic kidney disease: evaluation, classification, and stratification. Ann Intern Med. 2003;139:137-47.

6. Levey AS, Bosch JP, Lewis JB, Greene T, Rogers N, Roth D. A more accurate method to estimate glomerular filtration rate from serum creatinine: a new prediction equation. Modification of Diet in Renal Disease Study Group. Ann Intern Med. 1999;130:461-70.

7. Levey AS, Coresh J, Greene T, et al. Using standardized serum creatinine values in the modification of diet in renal disease study equation for estimating glomerular filtration rate. Ann Intern Med. 2006;145:247-54

8. Stevens LA, Coresh J, Feldman HI, et al. Evaluation of the modification of diet in renal disease study equation in a large diverse population. J Am Soc Nephrol. 2007:18:2749-57.

9. Levin A, Stevens PE. Summary of KDIGO 2012 CKD Guideline: behind the scenes, need for guidance, and a framework for moving forward. Kidney Int. 2014;85(1):49-61.

10. Andrassy KM. Comments on KDIGO 2012 Clinical Practice Guideline for the Evaluation and Management of Chronic Kidney Disease. Kidney Int. 2013;84:622-3.

11. Cockcroft DW, Gault MH. Prediction of creatinine clearance from serum creatinine. Nephron. 1976;16(1):31-41.

12. Shemesh $\mathrm{O}$, Golbetz $\mathrm{H}$, Kriss JP, Myers BD. Limitations of creatinine as a filtration marker in glomerulopathic patients. Kidney Int. 1985;28(5):830-8.

13. Levey AS, Perrone RD, Madias NE. Serum creatinine and renal function. Annu Rev Med. 1988;39:465-90.

14. Cartet-Farnier E, Goutelle-Audibert L, Maire P, De la Gastine B, Goutelle S. Implications of using the MDRD or CKD -EPI equation instead of the Cockcroft-Gault equation for estimating renal function and drug dosage adjustment in elderly patients. Fundam Clin Pharmacol. 2016. doi:10.1111/ fcp.12241. Ahead of print.

15. Dowling TC, Wang ES, Ferrucci L, Sorkin JD. Glomerular filtration rate equations overestimate creatinine clearance in older individuals enrolled in the Baltimore Longitudinal Study on Aging: impact on renal drug dosing. Pharmacotherapy. 2013:33(9):912-21.

16. Rostoker G, Andrivet P, Pham I, Griuncelli M, Adnot S. A modified CockcroftGault formula taking into account the body surface area gives a more accurate estimation of the glomerular filtration rate. J Nephrol. 2007;20(5):576-85.

17. Agence Nationale d'Accréditation et d'Evaluation en Santé. Diagnosis of adult chronic kidney failure. [In French]. Diabetes Metab. 2003;29(3):315-24.

18. Smilde TD, van Veldhuisen DJ, Navis G, Voors AA, Hillege HL. Drawbacks and prognostic value of formulas estimating renal function in patients with chronic heart failure and systolic dysfunction. Circulation. 2006;114:1572-80.

19. Zamora E, Lupon J, Vila J, et al. Estimated glomerular filtration rate and prognosis in heart failure: value of the Modification of Diet in Renal Disease Study-4, chronic kidney disease epidemiology collaboration, and CockcroftGault formulas. J Am Coll Cardiol. 2012;59:1709-15.

20. McAlister FA, Ezekowitz J, Tarantini $L$, et al. Renal dysfunction in patients with heart failure with preserved versus reduced ejection fraction: impact of the new Chronic Kidney Disease-Epidemiology Collaboration Group formula. Circ Heart Fail. 2012;5:309-14.

21. Plischke $M$, Neuhold $\mathrm{S}$, Kohl $\mathrm{M}$, et al. Renal function in heart failure: a disparity between estimating function and predicting mortality risk. Eur J Heart Fail. 2013:15(7):763-70.

22. Szummer K, Lundman P, Jacobson SH, et al. Cockcroft-Gault is better than the Modification of Diet in Renal Disease study formula at predicting outcome after a myocardial infarction: data from the Swedish Web-system for Enhancement and Development of Evidence-based care in Heart disease Evaluated According to Recommended Therapies (SWEDEHEART). Am Heart J. 2010;159:979-86.

23. Parsh J, Seth $\mathrm{M}$, Aronow $\mathrm{H}$, et al. Choice of estimated glomerular filtration rate equation impacts drug-dosing recommendations and risk stratification in patients with chronic kidney disease undergoing percutaneous coronary interventions. J Am Coll Cardiol. 2015;65(25):2714-23.

24. Jacobs $L$, Thijs $L$, Jin $Y$, et al. Heart 'omics' in AGEing (HOMAGE): design, research objectives and characteristics of the common database. J Biomed Res. 2014:28(5):349-59.

25. Staessen JA, Wang JG, Brand E, et al. Effects of three candidate genes on prevalence and incidence of hypertension in a Caucasian population. J Hypertens. 2001;19(8):1349-58. 
26. Mureddu GF, Agabiti N, Rizzello V, et al. Prevalence of preclinical and clinica heart failure in the elderly. A population-based study in Central Italy. Eur J Heart Fail. 2012;14(7):718-29.

27. Sever PS, Dahlof B, Poulter NR, et al. Rationale, design, methods and baseline demography of participants of the Anglo-Scandinavian Cardiac Outcomes Trial. ASCOT investigators. J Hypertens. 2001;19(6):1139-47.

28. Shepherd J, Blauw GJ, Murphy MB, et al. The design of a prospective study of Pravastatin in the Elderly at Risk (PROSPER). PROSPER Study Group. PROspective Study of Pravastatin in the Elderly at Risk. Am J Cardiol. 1999:84(10):1192-7.

29. Giorda CB, Cioffi G, de Simone G, et al. Predictors of early-stage left ventricular dysfunction in type 2 diabetes: results of DYDA study. Eur J Cardiovasc Prev Rehabil. 2011;18(3):415-23.

30. Seronde MF, Gayat E, Logeart D, et al. Comparison of the diagnostic and prognostic values of B-type and atrial-type natriuretic peptides in acute heart failure. Int J Cardiol. 2013;168(4):3404-11.

31. Brunner-La Rocca HP, Buser PT, Schindler R, Bernheim A, Rickenbacher P, Pfisterer M. Management of elderly patients with congestive heart failure-design of the Trial of Intensified versus standard Medical therapy in Elderly patients with Congestive Heart Failure (TIME-CHF). Am Heart J. 2006;151(5):949-55.

32. Dickstein K, Bebchuk J, Wittes J. The high-risk myocardial infarction database initiative. Prog Cardiovasc Dis. 2012;54:362-6.

33. Dargie HJ. Effect of carvedilol on outcome after myocardial infarction in patients with left-ventricular dysfunction: the CAPRICORN randomised trial. Lancet. 2001;357:1385-90.

34. Dargie HJ. Design and methodology of the CAPRICORN trial - a randomised double blind placebo controlled study of the impact of carvedilol on morbidity and mortality in patients with left ventricular dysfunction after myocardial infarction. Eur J Heart Fail. 2000;2:325-32.

35. Pitt $B$, Williams $G$, Remme W, et al. The EPHESUS trial: eplerenone in patients with heart failure due to systolic dysfunction complicating acute myocardial infarction. Eplerenone Post-AMI Heart Failure Efficacy and Survival Study. Cardiovasc Drugs Ther. 2001;15(1):79-87.

36. Pitt B, Remme W, Zannad F, et al. Eplerenone, a selective aldosterone blocker, in patients with left ventricular dysfunction after myocardial infarction. N Engl J Med. 2003;348:1309-21.

37. Dickstein K, Kjekshus J. Comparison of the effects of losartan and captopril on mortality in patients after acute myocardial infarction: the OPTIMAAL trial design. Optimal Therapy in Myocardial Infarction with the Angiotensin Il Antagonist Losartan. Am J Cardiol. 1999;83:477-81.

38. Dickstein K, Kjekshus J. Effects of losartan and captopril on mortality and morbidity in high-risk patients after acute myocardial infarction: the OPTIMAAL randomised trial. Optimal Trial in Myocardial Infarction with Angiotensin II Antagonist Losartan. Lancet. 2002;360:752-60.

39. Pfeffer MA, McMurray J, Leizorovicz A, et al. Valsartan in acute myocardial infarction trial (VALIANT): rationale and design. Am Heart J. 2000;140:727-50.

40. Pfeffer MA, McMurray JJ, Velazquez EJ, et al. Valsartan, captopril, or both in myocardial infarction complicated by heart failure, left ventricular dysfunction, or both. N Engl J Med. 2003;349:1893-906

41. Mosteller RD. Simplified calculation of body-surface area. N Engl J Med. 1987;317(17):1098.

42. von Lueder TG, Girerd N, Atar D, et al. Serum uric acid is associated with mortality and heart failure hospitalizations in patients with complicated myocardial infarction: findings from the High-Risk Myocardial Infarction Database Initiative. Eur J Heart Fail. 2015;17(11):1144-51.

43. Katz MH. Multivariable Analysis: A Practical Guide for Clinicians and Public Health Researchers. 3rd ed. Cambridge: Cambridge University Press; 2011.

44. Harrell Jr FE, Lee KL, Mark DB. Multivariable prognostic models: issues in developing models, evaluating assumptions and adequacy, and measuring and reducing errors. Stat Med. 1996;15:361-87.

45. Kang L, Chen W, Petrick NA, Gallas BD. Comparing two correlated C indices with right-censored survival outcome: a one-shot nonparametric approach. Stat Med. 2015:34(4):685-703.

46. Pencina MJ, D'Agostino Sr RB, D'Agostino Jr RB, Vasan RS. Evaluating the added predictive ability of a new marker: from area under the ROC curve to reclassification and beyond. Stat Med. 2008;27(2):157-72. discussion 207-112.

47. Uno H, Tian L, Cai T, Kohane IS, Wei LJ. A unified inference procedure for a class of measures to assess improvement in risk prediction systems with survival data. Stat Med. 2013:32(14):2430-42.
48. Coiro S, Rossignol P, Ambrosio G, et al. Prognostic value of residual pulmonary congestion at discharge assessed by lung ultrasound imaging in heart failure. Eur J Heart Fail. 2015;17(11):1172-81.

49. Rolin 3rd HA, Hall PM, Wei R. Inaccuracy of estimated creatinine clearance for prediction of iothalamate glomerular filtration rate. Am J Kidney Dis. 1984;4:48-54.

50. Sampson MJ, Drury PL. Accurate estimation of glomerular filtration rate in diabetic nephropathy from age, body weight, and serum creatinine. Diabetes Care. 1992;15(5):609-12.

51. Froissart M, Rossert J, Jacquot C, Paillard M, Houillier P. Predictive performance of the modification of diet in renal disease and CockcroftGault equations for estimating renal function. J Am Soc Nephrol. 2005;16:763-73.

52. Botev R, Mallie JP, Wetzels JF, Couchoud C, Schuck O. The clinician and estimation of glomerular filtration rate by creatinine-based formulas: current limitations and quo vadis. Clin J Am Soc Nephrol. 2011;6(4):937-50.

53. Poggio ED, Nef PC, Wang $X$, et al. Performance of the Cockcroft-Gault and modification of diet in renal disease equations in estimating GFR in ill hospitalized patients. Am J Kidney Dis. 2005;46:242-52.

54. Skali H, Uno H, Levey AS, Inker LA, Pfeffer MA, Solomon SD. Prognostic assessment of estimated glomerular filtration rate by the new Chronic Kidney Disease Epidemiology Collaboration equation in comparison with the Modification of Diet in Renal Disease Study equation. Am Heart J. 2011;162(3):548-54

55. Zafrir B, Salman N, Crespo-Leiro MG, et al. Body surface area as a prognostic marker in chronic heart failure patients: results from the Heart Failure Registry of the Heart Failure Association of the European Society of Cardiology. Eur J Heart Fail. 2016;18(7):859-68.

56. Levey AS, Inker LA, Coresh J. GFR estimation: from physiology to public health. Am J Kidney Dis. 2014;63(5):820-34.

\section{Submit your next manuscript to BioMed Central and we will help you at every step:}

- We accept pre-submission inquiries

- Our selector tool helps you to find the most relevant journal

- We provide round the clock customer support

- Convenient online submission

- Thorough peer review

- Inclusion in PubMed and all major indexing services

- Maximum visibility for your research

Submit your manuscript at www.biomedcentral.com/submit

) Biomed Central 